\title{
ESTUDO COMPARATIVO ENTRE AS TÉCNICAS DE PRECIPITAÇÃO POR REDUÇÃO QUÍMICA E RESINAS QUELANTES PARA RECUPERAÇÃO DE COBRE
}

\author{
Amilton Barbosa Botelho Junior ${ }^{1 *}$ \\ lara Alves Anes ${ }^{2}$ \\ Jorge Alberto Soares Tenório '
}

\section{Resumo}

O presente trabalho teve por objetivo comparar o uso da precipitação por redução química com o uso de resina quelante para a obtenção de cobre presente no lixiviado de níquel laterítico. Uma solução sintética foi preparada para simular o licor obtido na lixiviação do minério. $O$ processo de precipitação ocorreu através da redução do potencial redox da solução até $240 \mathrm{mV}$, e o agente redutor utilizado foi o ditionito de sódio. A velocidade de agitação também foi estudada na precipitação entre 0 e 200 rpm. A resina quelante Lewatit TP 207, de grupo funcional iminodiacetato, foi utilizada para os ensaios de troca-iônica. A solução foi posta em contato com a resina e submetida a agitação constante de $200 \mathrm{rpm}$. O efeito do $\mathrm{pH}$ foi estudado entre 0,50 e 2,00. Todos os ensaios foram realizados à $25^{\circ} \mathrm{C}$ e 120 min. Resultados indicam que o uso da técnica de precipitação por redução química foi mais vantajosa frente ao uso de resinas quelantes. Na redução química, foi possível obter uma precipitação seletiva de $96 \%$ do cobre em pH 0,50 e $45 \mathrm{rpm}$. Nos ensaios com resina quelante, foi possível adsorver $48 \%$ do cobre em pH 2,00 além de adsorver contaminantes como ferro e alumínio. Palavras-chave: Ditionito de sódio; Lewatit TP 207; Troca-iônica.

\section{COMPARATIVE STUDY BETWEEN TECHINIQUES OF PRECIPITATION BY CHEMICAL REDUCTION AND CHELATING RESINS FOR COPPER RECOVERY}

\begin{abstract}
The goal of this work was to compare the use of precipitation by chemical reduction with the use of chelating resin to obtain copper present in nickel laterite leach solution. A synthetic solution was prepared to simulate the liquor obtained in ore leaching. The precipitation process was performed through reducing the redox potential of solution until $240 \mathrm{mV}$, and the reducing agent used was sodium dithionite. The effect of stirring speed was also studied for precipitation between 0 and $200 \mathrm{rpm}$. The chelating resin Lewatit TP 207, of functional group iminodiacetate, was used for ion exchange experiments. The solution was put in contact with the resin and taken a constant stirring in $200 \mathrm{rpm}$. The effect of pH was studied between 0.50 and 2.00 . All experiments were performed at $25^{\circ} \mathrm{C}$ and $120 \mathrm{~min}$. Results indicated that the use of precipitation technique by chemical reduction was more advantageous than chelating resins. In chemical reduction, it was possible to obtain a selective precipitation of $96 \%$ of copper at $\mathrm{pH} 0.50$ and $45 \mathrm{rpm}$. In experiments using chelating resins, it was possible to absorb $48 \%$ of copper at $\mathrm{pH} 2.00$, and also contaminants such as iron and aluminum.
\end{abstract}

Keywords: Sodium dithionite; Lewatit TP 207; lon-exchange.

\section{INTRODUÇÃO}

No processo hidrometalúrgico, após a etapa de lixiviação do minério, têm-se etapas de purificação e separação para remoção de impurezas. Isto se deve, pois, impurezas também são lixiviadas junto com o metal de interesse [I].
No caso do minério laterítico de níquel, entre estas etapas encontram-se a extração por solventes, precipitação e a troca-iônica por meio de resinas [2]. A técnica de extração por solventes é comumente utilizada no processo extrativo

'Departamento de Engenharia Química, Escola Politécnica, Universidade de São Paulo - USP, São Paulo, SP, Brasil. E-mail: amilton.junior@usp.br ${ }^{2}$ Escola Superior de Química, Faculdades Oswaldo Cruz, São Paulo, SP, Brasil. 
para separação de cobalto da solução rica em níquel [2]. Aliprandini estudou a separação de metais do lixiviado de níquel laterítico utilizando a extração por solventes. Na primeira etapa, estudou-se a remoção do ferro utilizando o extratante Cyanex 272. Contudo, nesta etapa, houve perda de cobre e cobalto por arraste. $\mathrm{Na}$ etapa seguinte, foi estudado o extratante Acorga M5640, onde foi possível demonstrar que é possível separar todo o cobre da solução [3].

Para o uso da técnica de precipitação por meio de hidróxidos, Jiménez Correa et al. [4] mostrou que a remoção de ferro é possível em $\mathrm{pH}$ acima de 2,00. Porém, ocorre também a co-precipitação de cobre e cobalto. Botelho et al. [5] mostrou ser possível a precipitação de cobre utilizando ditionito de sódio, reduzindo o potencial da solução até $240 \mathrm{mV}$. Porém, não foram estudadas variáveis como $\mathrm{pH}$ e temperatura na precipitação.

No caso da técnica de troca-iônica por resinas quelantes, Littlejohn \& Vaughan verificou-se que, a partir de um lixiviado de níquel laterítico, a resina quelante Lewatit TP 207, de grupo funcional iminodiacetato, foi mais seletiva para cobre dentre as resinas estudadas. Contudo, os resultados mostram que outros metais também foram adsorvidos pela resina [6].

Desta forma, o presente trabalho teve por objetivo comparar o uso da técnica de precipitação por redução química com a técnica de troca-iônica utilizando resinas quelantes. Para reduzir o potencial da solução, utilizou-se o ditionito de sódio I mol/L como agente redutor. A velocidade de agitação na precipitação também foi estudada. Para os ensaios de troca-iônica foi utilizada a resina quelante Lewatit TP 207. Os ensaios foram realizados em batelada a $25^{\circ} \mathrm{C}$, sob agitação constante. Amostras foram analisadas por Espectroscopia de raios $X$ por dispersão em energia (EDX).

\section{MATERIAIS E MÉTODOS}

\section{I Materiais}

Solução sintética foi preparada a fim de simular a composição do licor de níquel laterítico após a etapa de lixiviação, conforme apresentado na Tabela I. Sulfatos de cada metal foram dissolvidos em água deionizada e o
$\mathrm{pH}$ corrigido até 0,50 para equilibrar a concentração de íons sulfato. A resina catiônica quelante escolhida para os ensaios de troca iônica foi a Lewatit TP 207, de grupo funcional quelante iminodiacetato. Esta resina possui matriz de poliestireno com ligação cruzada, $\mathrm{pH}$ de trabalho entre 0,00 e 14,00 , densidade de $1,17 \mathrm{~g} / \mathrm{mL}$ [7]. Esta resina é seletiva para adsorção de cobre frente aos outros metais presentes na solução [8]. Para os ensaios de precipitação por redução de potencial redox, preparou-se uma solução de ditionito de sódio $1 \mathrm{~mol} / \mathrm{L}$, e o potencial foi reduzido até $240 \mathrm{mV}[5,9]$.

\subsection{Metodologia}

\subsection{Ensaios de precipitaçao}

Nos ensaios de precipitação por redução química, a solução de ditionido de sódio foi adicionada na solução sintética em um béquer sob agitação magnética lenta até que o potencial redox fosse reduzido de $900 \mathrm{mV}$ para $240 \mathrm{mV}$. A mistura foi então colocada em um shaker orbital sob agitação à $25^{\circ} \mathrm{C}$ durante $\mathrm{I} 20 \mathrm{~min}$ (Figura I). A velocidade de agitação foi estudada entre 0 e $200 \mathrm{rpm}$ e o efeito do $\mathrm{pH}$ entre 0,50 e 2,00.

Após o tempo de reação, o precipitado foi filtrado utilizando-se filtro para filtração lenta (retenção nominal: $2 \mu \mathrm{m}$ ). O precipitado foi seco por 24 horas em estufa à $60^{\circ} \mathrm{C}$ e analisado em Microscopia Eletrônica de Varredura acoplado a Espectroscopia de Raios-X (MEV-EDS - Phenom modelo proX).

\subsubsection{Ensaios de troca-iônica}

Antes dos ensaios de troca iônica, a resina foi lavada utilizando ácido clorídrico $6 \mathrm{~mol} / \mathrm{L}$ e hidróxido de sódio $2 \mathrm{~mol} / \mathrm{L}$ em três etapas intercaladas, e utilizando água deionizada entre cada lavagem. Após a lavagem, ácido sulfúrico I mol/L foi utilizado para condicionar a resina. A resina foi então

Tabela I. Concentração em $\mathrm{mg} / \mathrm{L}$ dos metais presentes na solução sintética

\begin{tabular}{lccccccccc}
\hline Metais & $\mathrm{Al}$ & $\mathrm{Co}$ & $\mathrm{Cu}$ & $\mathrm{Cr}$ & $\mathrm{Fe}$ & $\mathrm{Mg}$ & $\mathrm{Mn}$ & $\mathrm{Ni}$ & $\mathrm{Zn}$ \\
\hline $\begin{array}{l}\text { \% em } \\
\text { massa }\end{array}$ & 12,11 & 0,23 & 0,43 & 0,58 & 55,24 & 22,95 & $\mathrm{I}, 17$ & 7,19 & $0,1 \mathrm{I}$ \\
\hline
\end{tabular}
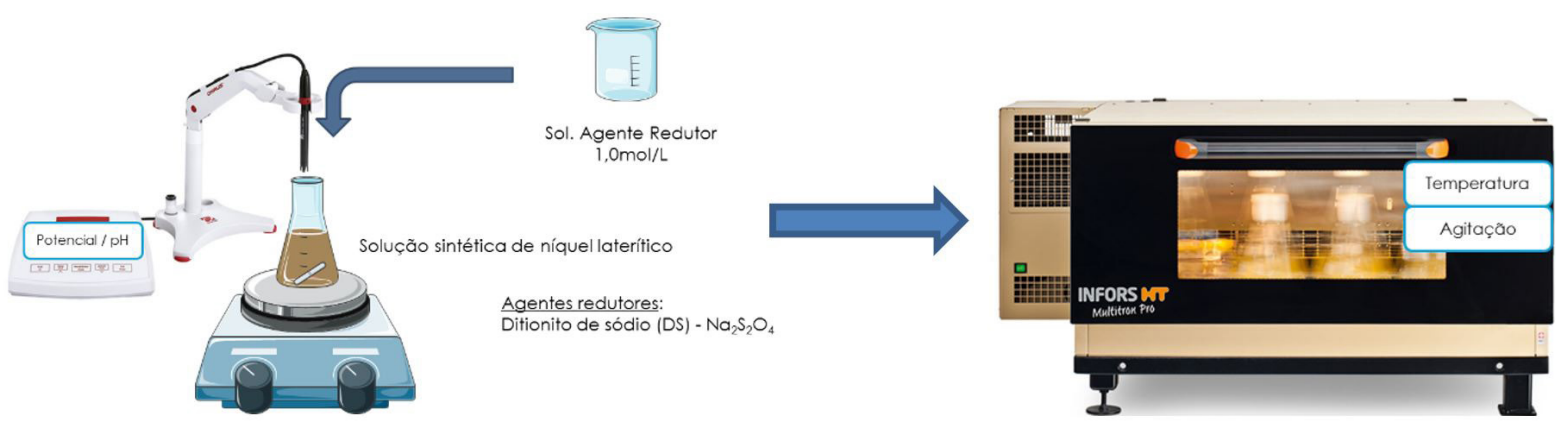

Figura I. Esquema dos ensaios de precipitação de cobre por redução de potencial. 
filtrada e seca em estufa a $60^{\circ} \mathrm{C}$ por 24 horas [10]. Para os ensaios de troca iônica, I $\mathrm{mL}$ de resina foi utilizada para cada $100 \mathrm{~mL}$ de solução em erlenmeyer de $250 \mathrm{~mL}$ sob agitação de $200 \mathrm{rpm}$ à $25^{\circ} \mathrm{C}$. O efeito do $\mathrm{pH}$ foi estudado entre 0,50 e 2,00. As Equações I e 2 foram utilizadas para quantificar os metais adsorvidos pela resina (Equação I) e a porcentagem de íons adsorvidos (Equação 2) [II, I2],

$$
\begin{aligned}
& q_{t}=\left(C_{0}-C_{t}\right) \times \frac{v}{m} \\
& \% S=\frac{\left(C_{0}-C_{t}\right)}{C_{0}} \times 100 \%
\end{aligned}
$$

onde: $\mathrm{q}_{\mathrm{t}}=$ capacidade da adsorvida no equilíbrio em massa de íon metálico por massa de resina $(\mathrm{mg} / \mathrm{g}) ; \mathrm{C}_{0}$ e $C_{t}=$ concentrações dos íons no tempo 0 e no tempo $\mathrm{t}(\mathrm{mg} / \mathrm{L}) ; \mathrm{V}=$ volume do licor $(\mathrm{L}) ; \mathrm{m}=$ massa da resina $(\mathrm{g})$.

$\mathrm{O} \mathrm{pH}$ foi medido utilizando eletrodo $\mathrm{Ag} / \mathrm{AgCl}$ (Sensoglass) e o potencial utilizando eletrodo redox $\mathrm{Ag} / \mathrm{AgCl}$, no qual somou-se $240 \mathrm{mV}$ para comparar o valor medido com o Diagrama de Pourbaix do cobre (SHE) [I]. As análises das amostras antes e após cada processo foram feitas utilizando a técnica de Espectroscopia de raio X (EDX).

\section{RESULTADOS E DISCUSSÃO}

\section{I Efeito da Velocidade de Agitação}

Para o processo de precipitação do cobre por meio da redução química, o efeito da velocidade de agitação foi estudado entre 0 e $200 \mathrm{rpm}$ em pH 0,50. Os resultados obtidos estão apresentados na Tabela 2. A velocidade de agitação influenciou na precipitação do cobre.

Tabela 2. Porcentagem de precipitação de cobre para diferentes velocidades de agitação

\begin{tabular}{cccccc}
\hline $\begin{array}{l}\text { Porcentagem de } \\
\text { precipitação (\%) }\end{array}$ & $\mathbf{9 4 , 4 7}$ & $\mathbf{9 4 , 9 5}$ & $\mathbf{9 3 , 4 2}$ & $\mathbf{8 5 , 4 5}$ & $\mathbf{7 8 , 7 3}$ \\
\hline Velocidade de agitação (rpm) & 0 & 45 & 90 & 150 & 200 \\
\hline
\end{tabular}

Em 0 e 45 rpm, observou-se precipitações de 94,47\% e 94,95\%, respectivamente,

Para o aumento da velocidade de agitação para 90 rpm, obteve-se $93,43 \%$ de precipitação de cobre. Para as velocidades de agitação $150 \mathrm{rpm}$ e $200 \mathrm{rpm}$, as porcentagens de precipitação foram de $85,45 \%$ e $78,73 \%$, respectivamente. $O$ precipitado obtido é $77 \%$ na forma de sulfeto de cobre, e $23 \%$ na forma de $\mathrm{S}_{8}$ [5]. Desta forma, para o estudo do efeito do $\mathrm{pH}$ utilizou-se a velocidade de agitação de $45 \mathrm{rpm}$.

Uma das hipóteses que pode ser levantada destes resultados, é que o oxigênio presente no ar age como agente oxidante no processo. Verificou-se que o potencial da solução aumentou - após os 120 min de reação - com o aumento da velocidade de agitação.

Botelho et al. [9] estudou o efeito da velocidade de agitação (0-200 rpm) para o processo de redução do $\mathrm{Fe}$ (III) para $\mathrm{Fe}$ (II) utilizando ditionito de sódio. A solução estudada tinha mesma composição do que a apresentada na Tabela I. Neste caso, não foi observada diferença entre as velocidades estudadas. Silva et al. [13] estudou o mesmo processo de redução aplicado ao minério caulim, onde verificou-se que a melhor velocidade de agitação foi de $90 \mathrm{rpm}$. Este valor ideal para esta aplicação se deve para diminuir a ação do oxigênio presente no ar como agente oxidante. Conforme resultados obtidos e apresentados na Tabela 2 , a velocidade de agitação ideal para precipitação do cobre é de $45 \mathrm{rpm}$. Esta velocidade foi adotada no estudo do efeito do $\mathrm{pH}$.

\subsection{Efeito do pH na Recuperação de Cobre}

$\mathrm{O}$ efeito do $\mathrm{pH}$ foi estudado entre $\mathrm{pH} 0,50$ e 2,00. Os ensaios de troca-iônica foram realizados em batelada. Os ensaios de precipitação redução química foram realizados a uma agitação de $45 \mathrm{rpm}$. Os ensaios decorreram ao longo de 120 min a $25^{\circ} \mathrm{C}$. Figura 2 apresenta os resultados obtidos para porcentagem de recuperação de cobre utilizando as duas técnicas. Para a técnica de precipitação por redução química, menores valores de $\mathrm{pH}$ aumentam a eficiência no processo de recuperação de cobre. Em pH 0,50 foi possível obter $94,95 \%$ de cobre, mantendo-se nesse valor em pH I,00 e atingindo-se $53,03 \%$ a recuperação de cobre

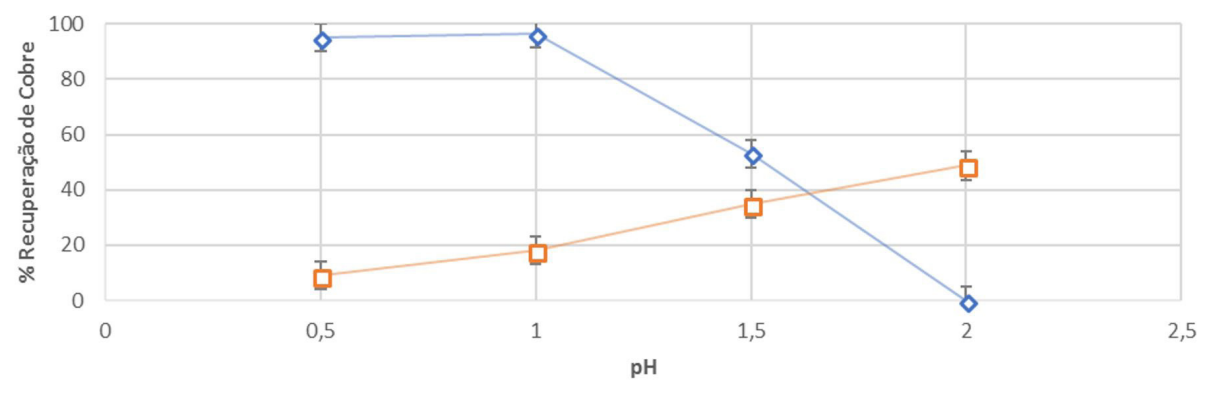

—-Redução Química $\quad$ - —-Troca iônica

Figura 2. Resultados para recuperação de cobre utilizando a técnica de troca-iônica e precipitação por redução química. 
em pH I,50. Não foi possível recuperar o cobre por meio desta técnica em $\mathrm{pH} 2,00$.

Para o uso da técnica de troca-iônica, a recuperação de metais é favorecida quando se diminui a concentração de íons $\mathrm{H}^{+}$presentes na solução, isto é, quando se aumenta o $\mathrm{pH}$ da solução. Durante o aumento do $\mathrm{pH}$, ocorre a desprotonação do grupo funcional da resina, aumentando assim a quantidade de sítios ativos disponíveis [1 I, 14]. Em pH 2,00, foi possível recuperar $48,72 \%$ do cobre presente na solução. Para a solução estudada, há um limite no qual se pode aumentar o $\mathrm{pH}$ devido à presença de ferro. $\mathrm{Em} \mathrm{pH}$ acima de 2,00, o ferro precipita em forma de hidróxidos junto com cobre e cobalto (co-precipitação) [4].

De acordo com o Diagrama de Pourbaix (Figura 3), para um mesmo potencial $-240 \mathrm{mV}$ - e variando o $\mathrm{pH}$ entre 0,50 e 2,00, a substância a ser formada vai mudando. $\mathrm{Em} \mathrm{pH} \mathrm{2,00} \mathrm{e} \mathrm{potencial} \mathrm{de} 240 \mathrm{mV}$ o cobre está na região de íon solúvel, enquanto que em pH 0,50 e I,00 o cobre está na região de formação de sulfeto de cobre [5]. Análises no MEV-EDS indicaram a presença de cobre e enxofre no precipitado obtido.

Além disso, em pH 0,50 e reduzindo o potencial da solução até $240 \mathrm{mV}$, foi possível uma precipitação seletiva de cobre. Na recuperação de cobre utilizando a técnica de troca-iônica outros metais foram adsorvidos. A Tabela 3 apresenta a porcentagem de metais adsorvidos pela resina para cada valor de $\mathrm{pH}$ estudado. Em todos as condições estudadas, outros metais também foram adsorvidos pela resina. Isto ocorre, pois, a resina é seletiva para cobre, mas não impede que outros metais possam ser adsorvidos. Neste caso, a adsorção é menor em comparação com o cobre. Para obter um produto com maior pureza, etapas de purificação ainda seriam necessárias.

\section{CONCLUSÃO}

O presente trabalho teve por objetivo comparar as técnicas de precipitação por redução química com a troca-iônica utilizando resinas quelantes. A velocidade de agitação na precipitação química e o efeito do $\mathrm{pH}$ para as duas técnicas foi estudado. A velocidade de agitação ideal para precipitação de cobre foi de $45 \mathrm{rpm}$. Em pH 0,50, foi possível precipitar $96 \%$ do cobre presente na solução. Para os ensaios utilizando resina quelante Lewatit TP 207, em $\mathrm{pH} 2,00$ foi possível adsorver $49 \%$ deste íon. Os resultados

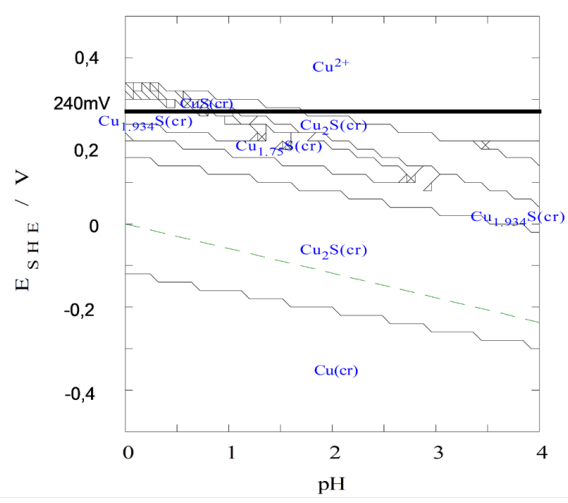

Figura 3. Diagrama de Pourbaix do sistema Cu-S- $\mathrm{H}_{2} \mathrm{O}$ nas condições em que os ensaios foram realizados.

Tabela 3. Porcentagens de metais adsorvidos pela resina quelante durante os ensaios de troca-iônica

\begin{tabular}{ccrrr}
\hline Metais & $\mathbf{0 , 5 0}$ & $\mathbf{I , 0 0}$ & $\mathbf{I , 5 0}$ & \multicolumn{1}{c}{$\mathbf{2 , 0 0}$} \\
\hline $\mathrm{Al}$ & $0,12 \%$ & $4,68 \%$ & $0,00 \%$ & $\mathbf{3 , 4 3} \%$ \\
$\mathrm{Co}$ & $\mathrm{I}, 25 \%$ & $2,14 \%$ & $3,69 \%$ & $\mathbf{3 , 0 3} \%$ \\
$\mathrm{Cr}$ & $1,31 \%$ & $0,00 \%$ & $1,29 \%$ & $\mathbf{0 , 0 0 \%}$ \\
$\mathrm{Cu}$ & $8,94 \%$ & $17,95 \%$ & $34,78 \%$ & $\mathbf{4 8 , 7 2 \%}$ \\
$\mathrm{Fe}$ & $2,77 \%$ & $\mathrm{I}, 02 \%$ & $2,39 \%$ & $\mathbf{I , 7 8 \%}$ \\
$\mathrm{Mg}$ & $0,00 \%$ & $5,14 \%$ & $0,43 \%$ & $\mathbf{3 , 7 3} \%$ \\
$\mathrm{Mn}$ & $0,43 \%$ & $0,00 \%$ & $1,84 \%$ & $\mathbf{I , 8 2} \%$ \\
$\mathrm{Ni}$ & $2,06 \%$ & $0,03 \%$ & $1,78 \%$ & $\mathbf{I , 8 2} \%$ \\
$\mathrm{Zn}$ & $5,03 \%$ & $0,00 \%$ & $2,70 \%$ & $\mathbf{6 , 1 4 \%}$ \\
\hline
\end{tabular}

apresentados sugerem que a técnica de precipitação é mais vantajosa, uma vez que foi possível separar apenas cobre da solução. No uso da resina quelante Lewatit TP 207, outros metais também foram adsorvidos pela resina. Contudo, o uso do ditionito de sódio como agente redutor é perigoso, devido à formação de sulfeto de hidrogênio em $\mathrm{pH}$ ácido. Estudos futuros podem verificar a aplicabilidade de outros agentes redutores para precipitação seletiva do cobre.

\section{Agradecimentos}

À Fundação de Amparo à Pesquisa do Estado de São Paulo e à CAPES (FAPESP - $n^{\circ} 2012 / 5$ I87I-9, $n^{\circ}$ 2016/05527-5 e $\mathrm{n}^{\circ} 2017 / 17340-0$ ) pelo suporte financeiro.

\section{REFERÊNCIAS}

I Havlik T. Hydrometallurgy: principles and application. Hydrometallurgy. 200 I;6I (3):229-230. http://dx.doi. org/I0.1016/S0304-386X(0I)00178-5.

2 Crundwell FK, Moats MS, Ramachandran V, Robinson TG, Davenport WG. Extractive metallurgy of nickel, cobalt and platinum-group metals. Oxford: Elsevier; 201 I. 583 p. [acesso em 29 maio 2019]. Disponível em: http:// linkinghub.elsevier.com/retrieve/pii/B97800809680941000I2 
3 Aliprandini P. O uso da extração por solventes para tratamento de licor de lixiviação de minério limonítico de níquel [dissertação]. Universidade de São Paulo; 20 I7. http://dx.doi.org/I0.I I606/D.3.20I7.tde-230220I7-II 0 I09.

4 Jiménez Correa MM, Aliprandini P, Soares Tenório JA, Romano Espinosa DC. Precipitation of metals from liquor obtained in nickel mining. In: Rewas 2016 Towar Mater Resour Sustain. Wiley; 2016. p. 333-338. http://dx.doi. org/10.1002/978III 9275039.ch52.

5 Botelho Junior AB, Anes IA, Carvalho MA, Espinosa DCR, Tenório JAS. Recovery of copper from nickel laterite leach waste by chemical reduction using sodium dithionite. In: Energy technology 2018. Ist ed. Arizona: The Minerals, Metals \& Materials Society; 2018. p. 429-434. http://dx.doi.org/I0.1007/978-3-319-72362-4_38.

6 Littlejohn P, Vaughan J. Selectivity of commercial and novel mixed functionality cation exchange resins in mildly acidic sulfate and mixed sulfate-chloride solution. Hydrometallurgy. 20I2; I2I-124:90-99. http://dx.doi.org/I0.1016/j. hydromet.2012.04.00I.

7 Lanxess. Product Information: Lewatit ${ }^{\circledR}$ TP 207. 20I I. p. I-5 [acesso em 29 maio 2019]. Disponível em: http:// www.lenntech.com/Data-sheets/Lewatit-TP-207-L.pdf

8 Botelho Junior A.B., Jiménez Correa M.M., Espinosa, D.C.R., Tenório, J.A.S. Study of the reduction process of iron in leachate from nickel mining waste. Brazilian Journal of Chemical Engineering. 2018; 35: I24I-1248. https://doi. org/10.1590/0104-6632.20180354s20170323.

9 Botelho AB Jr, Jiménez Correa MM, Espinosa DCR, Tenório JAS. Study of reducing process of iron in leachate from nickel mining waste. Brazilian Journal of Chemical Engineering. 2018;35:124I-I 248.

10 Abrão A. Operações de troca iônica. São Paulo: Instituto de Pesquisas Energéticas e Nucleares - CNEN/SP; 20 I4. $201 \mathrm{p}$.

I I Rudnicki P, Hubicki Z, Kołody ska D. Evaluation of heavy metal ions removal from acidic waste water streams. Chemical Engineering Journal. 2014;252:362-373. http://dx.doi.org/I0.1016/j.cej.2014.04.035.

12 Yu Z, Qi T, Qu J, Wang L, Chu J. Removal of $\mathrm{Ca}$ (II) and $\mathrm{Mg}$ (II) from potassium chromate solution on Amberlite IRC 748 synthetic resin by ion exchange. Journal of Hazardous Materials. 2009;167(I-3):406-4I2. http://dx.doi. org/10.1016/j.jhazmat.2008.12.140.

I3 Silva FANG, Garrido FMS, Medeiros ME, Sampaio JA, Luz AB, Mello LS, et al. Alvejamento químico de caulins brasileiros: efeito do potencial eletroquímico da polpa e do ajuste do pH. Quimica Nova. 20I I;34(2):262-267. http://dx.doi.org/10.1590/S0100-4042201 10002000I7.

I4 Zainol Z, Nicol MJ. Ion-exchange equilibria of $\mathrm{Ni2}+, \mathrm{Co} 2+, \mathrm{Mn2}+$ and $\mathrm{Mg} 2+$ with iminodiacetic acid chelating resin Amberlite IRC 748. Hydrometallurgy. 2009;99(3-4): 175-I80. http://dx.doi.org/I0.1016/j. hydromet.2009.08.004.

Recebido em: 06 Jul. 2018

Aceito em: 15 Jan. 2019 